\title{
Total knee arthroplasty—what do we know and what don't
}

\author{
R. Becker
}

Published online: 20 May 2011

(C) Springer-Verlag 2011

The number of publications dealing with total knee arthroplasty (TKA) is constantly rising. There were 454 publications in 2000, 849 publications in 2005 and already 1,378 publications in 2010 in PubMed. KSSTA has published 70 papers about the TKA focusing on biomechanical studies and clinical outcome studies dealing with different type of implants. There is an intense ongoing discussing about the best surgical techniques in order to achieve equal extension and flexion.

According to the study by Classen et al. [1], the tibial first technique, which is rather a soft tissue orientated technique, seems to work well in order to balance the knee in both flexion and extension. It remains questionable whether this technique might be superior in comparison with the referenced resection technique or the combination of both the referenced resection technique for the extension gap and the gap balance technique for the flexion gap. The aim is to achieve a well-balanced knee throughout the range of motion.

How precise are we in detecting our anatomical landmarks and how precise can we perform our bone cuts? Does the usage of a tourniquet during surgery show any impact on the outcome? How do we manage pain control after surgery and what seems to be the safest way of adequate antithrombotic management. What about patient's expectation in regard to TKA? Does it have any effect on our outcome? What we know, most of the patients can be successfully treated with TKA. However, there seems to be a certain number, which is not completely happy after

\section{R. Becker $(\square)$}

Department of Orthopaedic and Traumatology, City-Hospital Brandenburg, Hochstrasse 29, 14770 Brandenburg/Havel, Germany

e-mail: roland_becker@yahoo.de surgery. Theses are the patients, which we have to take care off. We have to analyse very carefully why these patients are unhappy. Are the symptoms directly related to your surgery or are there patient-related factors also? Based on extensive clinical and laboratory studies and the experience of the surgeons, we might be able to improve our outcome after TKA further.

The current issue presents a meta-analysis about the usage of the tourniquet in TKA. Review papers are always welcome, but the analysis has to be done meticulous. These papers provide a nice summary about a certain subject and they inform us about the level of evidence available in the current literature. There are only eight randomized control trials dealing with the usefulness of a tourniquet during TKA surgery. The usage of the tourniquet will reduce the surgical time, but we are not able to reduce the total blood loss. However, there might be an increased risk of thromboembolic complication. Matziolis et al. [3] looked at the endothelin-1 (ET-1), a mediator for disturbance of the microcirculation, and found a 3.3 times increase when the tourniquet was used and 2.9 times increase w/o a tourniquet. The peak level was found $6 \mathrm{~h}$ after surgery. However, he concluded that the soft tissue damage during surgery might have more significant impact on the ET- 1 than the usage of the tourniquet.

Furthermore, you will find a paper about the femoral component rotation in TKA, which is an ongoing discussion between the surgeons. Malrotation of the femoral component seems to be a common problem in TKA causing persistent pain and dysfunction [2]. What is the best technique in order to position the femoral component correctly? Femoral component rotation effect the patella tracking as already reported by Rodhas et al. [5] 20 years ago. He recommended a more external position in order to bring the patellar tracking closer to the intact knee. 
Another interesting paper of the current issue deals with wound complication after TKA [4]. Nobody likes severe wound complications after surgery. However, sometimes one may have to solve the problem. The authors point out that there is still much controversy in how to deal with these patients, and there are no general guidelines available. Should we leave the implant in place or do we have to remove them, which will cause severe limitation of daily activity for our patients? Fasciocutaneous flaps or muscle flaps are successful in 75-100\%. Please read the informative paper, which will give you an excellent overview especially about the management of these very difficult cases.

The current issue includes different subjects about TKA, and we hope these papers are of interest for you. Some messages may influence your management or they might stimulate to reconsider certain aspects in your management. Sometimes, we may forget to be permanently critical with our routine work.

\section{References}

1. Classen T, Landgraeber S, Wegner A, Müller RD, von Knoch M (2010) Femoral component rotation in patients with leg axis deviation. Knee Surg Sports Traumatol Arthrosc (this issue). doi: 10.1007/s00167-010-1344-5

2. Czurda T, Fennema P, Baumgartner M Ritschl P (2009) The association between component malalignment and post-operative pain following navigation-assisted total knee arthroplasty: results of a cohort/nested case-control study. Knee Surg Sports Traumatol Arthrosc (this issue). doi:10.1007/s00167-009-0990-y

3. Matziolis G, Drahn T, Schröder JH, Krocker D, Tuischer J, Perka C (2005) Endothelin-1 is secreted after total knee arthroplasty regardless of the use of a tourniquet. J Orthop Res 23:392-396

4. Panni AS, Vasso M, Cerciello S, Salgarello M (2010) Wound complications in total knee arthroplasty. Which flap is to be used? With or without retention of prosthesis? Knee Surg Sports Traumatol Arthrosc (this issue). doi:10.1007/s00167-010-1328-5

5. Rhoads DD, Noble PC, Reuben JD, Mahoney OM, Tullos HS (1990) The effect of femoral component position on patellar tracking after total knee arthroplasty. Clin Orthop Relat Res 260:43-51 